Man lege jetzt für die $x$ (einschließich der $x^{p}$ ) eine Homologiebasis zugrunde, ebenso für die $y$ (einschließlich der $y^{p}$ ). Dann wird aus (5)

$$
z=\Sigma \alpha(x \cdot y)+\Sigma \beta\left[x^{p} y^{q}\right]+\Sigma x \cdot s+\Sigma y \cdot r+\mathfrak{r} m
$$

mit

$$
\left[x^{p} y^{q}\right]=p^{*}\left(x^{p} \cdot \bar{y}^{(q)}\right)-(-1)^{\varphi} q^{*}\left(x^{(p)} \cdot y^{q}\right) ;
$$

dabei sollen $p^{*}$ und $q^{*}$ teilerfremde Zahlen sein, die sich wie $p$ und $q$ verhalten, $\alpha$ soll alle ganzen Zahlen durchlaufen kzönnen und $\beta$ alle Reste $\bmod (p, q)$ (größter gem. Teiler von $p$ und $q$ ). Man beachte im Folgenden, daß wegen der Unabhängigkeit der $x^{p}$ die $\bar{x}^{(p)}$ unabhängig mod $X$ sind (analog für die $y^{p}$ ).

Sei nun $z$ ein Rand, also

$$
\Sigma \alpha(x \cdot y)+\Sigma \beta\left[x^{p} \cdot y^{q}\right]=\Sigma \mathfrak{r}(k \cdot l)
$$

die rechte Seite läßt sich dann auch schreiben mit mod $X$ unabhängigen $k^{\prime}$ und mod $Y$ unabhängigen $l^{\prime}$ :

$$
\sum\left(\mathfrak{r} k^{\prime} \cdot l^{\prime} \pm k^{\prime} \cdot \mathfrak{r} l^{\prime}\right)+\Sigma x \cdot s+\Sigma r \cdot y \cdot
$$

Vergleich mit der linken Seite liefert

$$
k^{\prime}=\Sigma \gamma \bar{x}^{(p)}, \quad l^{\prime}=\Sigma \delta \bar{y}^{(q)}, \quad r=0, \quad s=0,
$$

so daß die rechte Seite die Gestalt

annimmt.

$$
\sum \varepsilon \cdot\left(p x^{p} \cdot \bar{y}^{(q)} \pm q \bar{x}^{(p)} \cdot y^{q}\right)=\Sigma \varepsilon \cdot(p, q)\left[x^{p} y^{q}\right]
$$

Somit liefern die $x \cdot y$ und $\left[x^{p} y^{q}\right]$ aus (6) eine Basis der Bettischen Gruppe von $K \cdot L$, und da das direkte Produkt einer zyklischen Gruppe der Ordnung $p$ und einer der Ordnung $q$ eine der Ordnung $(p, q)$ ist, ist unsere Formel bewiesen.

\section{Die schlichten stetigen Bilder des Nullraums.}

Von

$$
\text { F. H a us dorff (Bonn). }
$$

Das Folgende ist eine kleine Ergänzung zu Herrn Kuratowski's 1) Theorie der schlichten, beiderseits Borelschen Abbildungen (homéomorphies de classe $\alpha, \beta$ ). Es ist dort festgestellt, dass jede Borelsche Menge $A$ eines separablen vollständigen Raumes schlichtes stetiges Bild einer im Nullraum $N$ abgeschlossenen Menge $N_{0}$ und, falls unabzählbar, nach Tilgung einer abzählbaren Menge $R$ schlichtes stetiges Bild von $N$ selbst ist; ausserdem wird die Klasse der inversen Abbildung präzisiert (vgl. unten (5)). Wenn wir fragen, wann $A$ schlichtes stetiges Bild von $N$ selbst ist (also $N_{0}=N$ oder $R=0$ angenommen werden kann), so ergibt sich als notwendige Bedingung, dass $A$ wie $N$ verdichtet sein muss; dies erweist sich aber auch als binreichend: die schlichten stetigen Bilder von $N$ sind mit den verdichteten Borelschen Mengen identisch ${ }^{2}$ ). Auch hierbei soll die Klasse der inversen Abbildung präzisiert werden (Satz I).

Wir stellen zunächst in (1) bis (7) einige Hilfsbetrachtungen und bekannte Resultate zusammen.

(1) Zur Abkürzung sollen die Borelschen Mengen eines metrischen Raumes nach dem Vorbild von H. Lebesgue so bezeichnet werden:

$$
\begin{aligned}
& F^{0}=F \text { abgeschlossen, } \\
& F^{\alpha}=\Pi_{n} G^{\xi n}
\end{aligned}
$$$$
\begin{aligned}
& G^{0}=G \text { offen, } \\
& G^{\alpha}=\sum F^{\xi_{n}}
\end{aligned}
$$

(für $\left.a>0, \xi_{n}<\alpha\right)$.

1) Wir zitieren die beiden Werke:

(A) Topologie I, Monografie Matematyczne, Warszawa-Lwów 1933.

(B) Sur une généralisation de la notion d'homéomorphie, Fund. Math. 22 (1934), p. 206-220.

2) Vgl. W. Sierpiński, Sur les images continues et biunivoques de l'ensemble de tous les nombres irrationnels, Mathematica 2 (1929), p. 18-21 (für lineare Borelsche Mengen). 
Also $F^{c}=$ ensemble de classe $\alpha$ multiplicative, $G^{c}=$ ensemble de classe $\alpha$ additive (Kuratowski); für die ersten Indizes:

$$
F^{1}=G_{\delta}, \quad F^{2}=F_{\sigma \delta}, \quad \ldots, \quad G^{1}=F_{\sigma}, \quad G^{2}=G_{\delta \sigma}, \quad \ldots
$$

Nachher, von (4) ab, sollen diese Borelschen Mengen immer in separablen vollständigen Räumen liegen, sodass z. B. $F$ einen separablen vollständigen Raum, $F^{1}=G_{\delta}$ einen separablen, topologisch vollständigen Raum bedeutet. Die Borelschen Mengen eines separablen, nicht notwendig vollständigen Raumes $A$ sind dann mit $A F^{\alpha}, A G^{\alpha}$ zu bezeichnen. Die Abbildung $f$ von $A$ auf $B$ heisst von der Klasse $\alpha$, wenn $f^{-1}\left(B F^{\prime}\right)$ stets ein $A F^{\alpha}$ ist; ist sie schlicht und $f^{-1}$ von der Klasse $\beta$, d.h. $f(A F)$ stets ein $B F^{\beta}$, so heisst $f$ von der Klasse $\alpha, \beta$ oder eine Abbildung $\alpha, \beta$.

(2) Der Bairesche Nullraum $N$ (mit der Menge der irrationalen Zahlen homöomorph) ist die Menge der natürlichen Zahlenfolgen $\lambda=\left(l_{1}, l_{2}, \ldots\right)$ mit der Metrik: ist $\mu=\left(m_{1}, m_{2}, \ldots\right) \neq \lambda$ und $k$ die kleinste Zahl mit $l_{k} \neq m_{k}$, so ist $|\lambda-\mu|=1 / k$; er wird hierdurch vollständig. Das Intervall $k$. Ordnung $N_{l_{1} \ldots l_{k}}$ ist die Menge der $\lambda$, deren erste $k$ Ziffern $l_{1}, \ldots, l_{k}$ sind; es ist in $N$ offen und abgeschlossen und ebenso wie sein Komplement mit $N$ homöomorph.

(3) Für eine Menge $A \subset X$ sei $\tilde{A}$ die Menge der Verdichtungspunkte, $A^{*}=A \widetilde{A}$ die Menge der in $A$ liegenden Verdichtungspunkte; eine Menge $A \subset \tilde{A}$ oder $A=A^{*}$ heisst verdichtet. Für eine verdichtete Menge $A$ ist die Bedingung $\widetilde{A}=\bar{A}$ notwendig and hinreichend; mit $A$ ist $\bar{A}$. verdichtet. Eine verdichtete Menge ist stets auch insichdicht; eine topologisch vollständige, insichdichte Menge ist auch verdichtet. Ist $X$ separabel, so sind, für jede Menge $A, \widetilde{A}$ und $A^{*}$ verdichtet, $A-A^{*}$ abzählbar.

Der Kürze halber sagen wir:

$$
A \text { ist in } O \text { verdichtet, wenn } A \subset C \subset \tilde{A} \text {, }
$$

d. h. wenn $A$ verdichtet und in $C$ dicht ist. Ist $A$ in $C$ verdichtet und $U$ offen, so ist $A U$ in $C U$ verdichtet. Ist $A \subset B \subset O$ und $A$ in $O$ verdichtet, so ist $A$ in $B$ und $B$ in $C$ verdichtet (auch umgekehrt).

Von jetzt an handelt es sich nur noch um Mengen in separablen vollständigen Räumen.

(4) Das Bild eines $F^{\alpha}(\alpha>0)$ bei einer Abbildung $\alpha, \beta$ ist ein $F^{\beta+\alpha}$ (A, p. 222). Das Bild eines $F$ bei einer Abbildung $0, \beta$ ist ein $F^{\beta+1}$.
Alles Folgende ist der Umkehrung der zweiten Behauptung von (4) gewidmet. Da diese Behauptung für $\beta=0$ gewiss umkehrbar ist (jedes $F^{\text {in }}$ ist mit einem $F$ homöomorph), nehmen wir $\beta>0$ an und schreiben $\alpha$ stati $\beta$.

(5) Jedes $F^{\alpha+1}(\alpha>0)$ entsteht durch eine Abbildung $0, \alpha$ aus einem 0-dimensionalen $F$ oder aus einer in $N$ abgeschlossenen Menge; falls unabzählbar, entsteht es, nach Weglassung einer abzählbaren Menge, aus $N$ selbst durch eine Abbildung $0, \alpha(\mathrm{B}, \mathrm{p} .215$, théorème 1 ; B, p. 216, corollaire 1; vgl. auch A, p. 224, théorème 2).

Beim Übergang von der ersten Behauptung (5) zur zweiten wird der folgende Satz von Herrn Mazurkiewicz verwendet (A, p. 225, théorème 3$)$ :

(6) Ist $X$ 0-dimensionales $F, A$ ein $G_{8}$ in $X, A$ und $X-A$ in $X$ dicht, so ist $A$ mit $N$ homöomorph.

Aus ihm folgern wir noch:

(7) Ist $A$ in $N$ dichtes $G_{\delta}$, so ist $A$ mit $N$ homöomorph.

Denn ist $X$ der dyadische Teilraum von $N$, aus den $\lambda=\left(l_{1}, l_{2}, \ldots\right)$ mit $l_{k}=1,2$ bestehend ( $X$ mit dem Cantorschen Diskontinuum homöomorph), so ist die Menge $X_{1} \subset X$ der $\lambda$ mit unendlich vielen $l_{k}=1$ mit $N$ homöomorph, $X_{2}=X-X_{1}$ abzählbar, beide Summanden in $X=X_{1}+X_{2}$ dicht. Ist $A$ in $X_{1}$ dichtes $G_{\delta}$, so auch in $X$, während zugleich $X-A \supset X_{2}$ in $X$ dicht ist; nach (6) ist $A$ mit $N$ homöomorph.

Unsere Absicht ist nun, die folgende Präzisierung von. (5) zu beweisen:

Satz I. Jedes verdichtete $F^{\alpha+1}(a>0)$ entsteht aus $N$ durch einè Abbildung 0,a.

Zunächst ist der Fall $\alpha=1$ zu behandeln, den wir besonders aussprechen:

Satz II. Jedes verdichtete $F^{2}=F_{\text {oo }}$ entsteht aus $N$ durch eine Abbildung 0,1.

Das ist eine (von Herrn Kuratowski selbst vermutete) Verschärfung des Satzes B, p. 210, wonach die verdichteten (=insichdichten) $I^{\prime}=G_{8}$ aus $N$ durch Abbildungen 0,1 entstehen.

Beim Beweise dieses Satzes wie auch von II spielt die Möglichkeit eine Rolle, eine insichdichte $F_{\sigma}$-Menge $B$ folgendermassen $z u$ zerlegen (der Raum $X$ ist bier zunächst noch beliebig): 


$$
\left\{\begin{array}{l}
B=D_{1}+D_{2}+\ldots \text { mit disjunkten Summanden } D_{l} \neq 0, \\
P_{l}=D_{1}+\ldots+D_{l} \text { perfekt. }
\end{array}\right.
$$

Wir nennen dies eine Zerlegung $Z$ and, wenn alle $D_{l}$ von Durchmessern $<\delta$ sind, eine Zerlegung $Z(\delta)$. Der Schwerpunkt der Forderung liegt übrigens im Nichtverschwinden aller $D_{l}$.

(8) Ist $X$ separabel, $B$ insichdicht und Differenz von zwei abgeschlossenen Mengen, so gestattet $B$ für jedes $\delta>0$ eine Zerlegung $\mathrm{Z}(\delta)$, bei der die $P_{l}=\overline{B U}_{l}\left(U_{l}\right.$ offen) sind.

(Lemme B, p. 208). Die besondere Form der $P_{l}$ ist von Herrn Kuratowski in den Wortlaut des Hilfssatzes nicht aufgenommen, aber aus dem Beweis ersichtlich. Es folgt daraus:

$\left(8^{*}\right)$ Ist $A$ in $B$ verdichtet, so ist jedes $A D_{l}$ in $D_{l}$ verdichtet.

Denn $A U_{l}$ ist in $B U_{l}$ verdichtet, also auch in $P_{l}\left(A U_{l}\right.$ ist verdichtet und in $B U_{l}$ oder in $P_{l}$ dicht); wegen $A U_{l} \subset A P_{l} \subset P_{l}$ ist $A P_{l}$ in $P_{l}$ verdichtet, und da $X-P_{l-1}$ offen ist, $A\left(P_{l}-P_{l-1}\right)$ in $P_{l}-P_{l-1}$ oder $A D_{l}$ in $D_{l}$ verdichtet.

Im Folgenden wird es sich auch bei Mengen $B=F_{\sigma}$ um solche Zerlegungen handeln, bei denen $A D_{l}$ in $D_{l}$ verdichtet ist; indessen kann man das nicht mehr, wie bei Mengen $B=F-F^{\prime}$, durch $P_{l}=\overline{B U_{l}}$ erreichen. (Es gibt insichdichte und sogar verdichtete $B=F_{\sigma}$, die überhaupt kein $\overline{B U} \neq 0$ mit offenem $U$ enthalten, z. B. wenn $B$ und $X-B$ in $X$ dicht sind).

(9) $\quad C \neq 0$ sei ein $F_{\sigma}$ (im separablen vollständigen Raume $X$ ) und $A$ eine in $C$ verdichtete analytische Menge. Dann gibt es für vorgeschriebenes $\delta>0$ eine Menge $B$ zwischen $A$ und $C(A \subset B C C)$ mit einer Zerlegung $Z(\delta)$, bei der jedes $A D_{l}$ in $D_{l}$ verdichtet ist.

Beweis. Man kann die abgeschlossenen Summanden von $C=\Sigma F_{h}$ von Durchmessern $<\delta$ annehmen. Wir setzen

$$
Q_{h}=\widetilde{A F}_{h}, \quad V_{h}=\left(A F_{h}\right)^{*}=A F_{h} \cdot Q_{h}=A Q_{h},
$$

sodass $A F_{h}-\nabla_{h}=R_{h}$ abzählbar ist; $V_{h}$ ist verdichtet, $Q_{h}$ perfekt, und da $V_{h} \subset Q_{h}=\widetilde{A F}_{h}=\widetilde{V}_{h}$, ist $V_{h}\left(=A Q_{h}\right)$ in $Q_{h}$ verdichtet. Sei $B=\Sigma\left(Q_{h}+R_{h}\right)$, so ist $A \subset B \subset C$, nämlich

$$
\Sigma A F_{h}=\Sigma\left(V_{h}+R_{h}\right) \subset \Sigma\left(Q_{h}+R_{h}\right) \subset \Sigma F_{h} .
$$

Die Menge $R=B-\Sigma Q_{h} \subset \Sigma R_{h} \subset A$ ist abzählbar und jedes $x \in R$ als Punkt der verdichteten analytischen Menge $A$ in einer perfekten
Menge $Q_{x} \subset A$ vom Durchmesser $<\delta$ enthalten, wobei natürlich $A Q_{x}=Q_{x}$ in $Q_{x}$ verdichtet ist; $R$ wird von abzählbar vielen $Q_{x}$ bedeckt, sagen wir $R \subset \Sigma Q_{k} \subset A \subset B$, also $B=\Sigma Q_{h}+R \subset \Sigma Q_{h}+\Sigma Q_{k} \subset B, B=\Sigma Q_{h}+\Sigma Q_{k}$.

Wir haben also jetzt $B=\Sigma Q_{l}$ mit perfekten Summanden von Durchmessern $<\delta$ derart, dass $A Q_{l}$ in $Q_{l}$ verdichtet ist; mit $D_{l}=Q_{l}-\left(Q_{1}+\ldots+Q_{l-1}\right)$ ist dann auch $A D_{l}$ in $D_{l}$ verdichtet, $D_{1}+\ldots+D_{l}=Q_{1}+\ldots+Q_{l}$ ist perfekt. Behalten wir nur die $D_{l} \neq 0$ bei und sind deren unendlich viele (die wir wieder $D_{1}, D_{2}, \ldots$ nennen), so ist unsere Behauptung bewiesen. Andernfalls aber ist $B$ perfekt und hat nach (8) eine Zerlegung $\mathrm{Z}(\delta)$ der verlangten Art.

(10) $A=C^{1} C^{2} \ldots$ sei ein verdichtetes $F_{\text {od }}$ (im separablen vollständigen Raume $X$ ), die $C^{k}$ Mengen $F_{\sigma}$. Dann lassen sich allen Komplexen $l_{1}, \ldots, l_{k}$ natürlicher Zahlen $(k=1,2, \ldots)$ Mengen $D_{l_{1} \ldots l_{k}}$ derart zuordnen:

(a) $\quad D_{l_{1} \ldots l_{k}}$ ist $\neq 0$ und vom Durchmesser $<1 / k ; A D_{l_{1} \ldots l_{k}}$ ist in $D_{l_{1} \ldots l_{k}}$ verdichtet.

( $\beta) \quad$ Die $D_{l_{1} \ldots l_{k}}$ sind bei festem $k$ disjunkt; $P_{l_{1} \ldots l_{k-1} l_{k}}=\sum_{l=1}^{l_{k}} D_{l_{1} \ldots l_{k-1} l}$ ist perfekt.

( $\gamma$ ) Für jede Folge $\lambda=\left(l_{1}, l_{2}, \ldots\right)$ ist

$$
D_{l_{1}} \supset D_{l_{1} l_{2}} \supset \ldots, \quad \text { also } \quad P_{l_{1}} \supset D_{l_{1}} \supset P_{l_{1} l_{2}} \supset D_{l_{1} l_{2}} \supset \ldots
$$

( $\delta) \quad$ Für $B^{k}=\sum_{l_{1} \ldots l_{k}} D_{l_{1} \ldots l_{k}}$ ist $A \subset B^{k} C C^{k}$.

Beweis. $A$ ist in $C^{\mathbf{2}} \bar{A}$ verdichtet; nach (9) gibt es eine Menge $B^{1}, A \subset B^{1} \subset C^{1} \bar{A}$, die eine Zerlegung $\mathrm{Z}(1)$ gestattet: $B^{1}=\Sigma D_{l}$ mit disjunkten $D_{l} \neq 0$ von Durchmessern $<1$ derart, dass $P_{l}=D_{1}+\ldots+D_{l}$ perfekt und $A D_{l}$ in $D_{l}$ verdichtet ist.

Nehmen wir an, die Mengen mit $k-1$ Indizes seien bereits bedingungsgemäss bestimmt; $\mu=l_{1}, \ldots, l_{k-1}$ bedeute einen $(k-1)$-gliedrigen Komplex und $\mu, l=l_{1}, \ldots, l_{k-1}, l$ den durch Hinzufügung von $l$ entstehenden $k$-gliedrigen. $A D_{\mu}$ ist in $D_{\mu} \neq 0$ verdichtet, also wegen $A D_{\mu} \subset C^{k} D_{\mu} \subset D_{\mu}$ auch $A D_{\mu}$ in $C^{k} D_{\mu}$, und die letzte Menge ist ein $F_{\sigma}$, da $D_{\mu}$ nach $(\beta)$ Differenz perfekter Mengen ist. Nach (9) gibt es eine Menge $B_{\mu}^{k}, A D_{\mu} \subset B_{\mu}^{k} \subset C^{k} D_{\mu}$, die eine $\mathrm{Z}(1 / k)$-Zerlegung $B_{\mu}^{k}=\sum_{I} D_{\mu l}$ (demnach $D_{\mu l} \subset D_{\mu}$ ) gestattet, bei der $A D_{\mu} \cdot D_{\mu l}=A D_{\mu l}$ in $D_{\mu l}$ verdichtet ist; die $D_{\mu l}$ sind disjunkt, $\neq 0$, von Durchmessern $<1 / k$, $P_{\mu l}=D_{\mu 1}+\ldots+D_{\mu l}$ perfekt. 
Aus $A D_{\mu} \subset \sum D_{\mu l} \subset C^{k}$ folgt durch Summation nach $\mu: A B^{k-1} \subset B^{k} \subset C^{k}$, d. h. $A \subset B^{k} \subset C^{k}$. Dadurch sind die Mengen mit $k$ Indizes bedingungs. gemäss bestimmt.

Auf Grund von $A \subset \Pi B^{k} \subset \Pi C^{k}=A$, also $A=\Pi B^{k}$ ist num II leicht zu beweisen. $Z u$ jedem $x \in A$ gibt es eine und nur eine Folge $\lambda=\left(l_{1}, l_{2}, \ldots\right)$ mit $x=D_{l_{1}} D_{l_{1} l_{2} \ldots}$; umgekehrt, weil $X$ vollständig ist und nach $(\gamma) P_{l_{1} \ldots l_{k}}$ für $\eta_{i}>1$ einen Durchmesser $<1 /(k-1)$ hat, gehört zu jeder Folge $\lambda$ ein Punkt $x=P_{l_{1}} P_{l_{1} l_{2}} \ldots=D_{l_{1}} D_{l_{1} l_{2}} \ldots \in A$. Hiermit ist eine schlichte Abbildung $x=f(\lambda)$ des Nullraums $N$ anf $A$ gegeben. Das Bild des Intervalls $N_{l_{1} \ldots l_{k}}$ ist $f\left(N_{l_{1} \ldots l_{k}}\right)=A D_{l_{1} \ldots l_{k}}$; beide Mengen haben Durchmesser $<1 / k$. Danach ist $f$ gleichmässig stetig; andererseits, da die $D$ Differenzen perfekter Mengen, also $F_{\sigma}$ sind und die Intervalle eine Basis der offenen Mengen in $N$ bilden, ist $f(G)=A F_{\sigma}=A G^{1}, \quad f$ von der Klasse 0,1 .

Alsdann beweisen wir I unter der Annahme $\alpha>1$ (die orst ganz zuletzt gebraucht wird; zunächst gilt alles auch nooh für $\alpha>0$ ).

Ist $B$ verdichtetes $F^{a+1}$, so gibt es nach (5) eine Zerlegung $B=A+R$, wo $A=f(N)$ vermöge einer Abbildung $0, a$ aus $N$ entsteht und $R=B-A$ abzählbar ist. Nun ist jeder Punkt $x \in R$ in einer perfekten Menge $Q_{2} \subset A+x$ enthalten, deren (in $N$ abgeschlossenes) Urbild $P_{x}=f^{-1}\left(Q_{x}\right)=f^{-1}\left(A Q_{x}\right)=f^{-1}\left(Q_{x}-x\right)$ nirgendsdicht ist. Denn für jede Umgebung $U$ von $x$ ist $A U$ (wie $B U$ ) unabzählbar, enthält also eine mit dem Cantorschen Diskontinuum $O$ homöomorphe Menge und, weil $C$ mit seinem „Quadrat" $(C, C)$ homöomorph ist, sogar $2^{\aleph_{0}}$ disjunkte perfekte Mengen $Q$; da von deren Urbildern $P=f^{-1}(Q)$ nur abzählbar viele einen inneren Punkt haben. können, enthält $A U$ ein perfektes $Q$ mit nirgendsdichtem Urbild $P$. Eine Folge solcher Umgebungen $U_{n}$ mit Durchmessern $\rightarrow 0$ liefert eine Folge perfekter $Q_{n} \subset A U_{n}$ mit nirgendsdichten Urbildern $P_{n}$ und dann ist $Q_{x}=\Sigma Q_{n}+x$ perfekt mit einem Urbild $P_{x}=\Sigma P_{n}$, das in $N$ abgeschlossen und (von I. Kategorie, also) nirgendsdicht ist. Macht man dies für jedes $x \in R$ und setzt $Q=\Sigma Q_{x}, P=f^{-1}(Q)=\Sigma P_{x}$, so ist $R \subset Q \subset A+R$ und

$$
f(P)=A Q=Q-R, \quad f(N-P)=A-(Q-R)=B-Q .
$$

Die Teilfunktion $f \mid N-P$ ist von der Klasse $0, \alpha$ und, da $P$ ein $F_{\sigma}$ von 1 . Kategorie, $N-P$ ein in $N$ dichtes $G_{\delta}$ und nach (7) mit $N$ homöomorph ist, haben wir

$$
B-Q=g_{1}\left(N_{1}\right),
$$

wo $N_{1}$ etwa ein Intervall von $N$ und $g_{1}$ von der Klasse $0, \alpha$ ist. Sodann ist $Q$ als Summe abzählbar vieler perfekter Mengen ein verdichtetes $F_{\sigma}$ und entsteht nach II (übrigens ist dieser Spezialfall natürlich viel schneller zu beweisen) aus $N$ durch eine Abbildung 0,1 ; da $N_{2}=N-N_{1}$ wieder mit $N$ homöomorph ist, sei

$$
Q=g_{2}\left(N_{2}\right) \text {, }
$$

$g_{2}$ von der Klasse 0,1 . Wir haben jetzt $\left(g=g_{1}, g_{2}\right.$ in $\left.N_{1}, N_{2}\right)$

$$
B=g(N),
$$

$g$ ist schlicht und stetig. Ist $F$ in $N$ abgeschlossen, so ist $g_{1}\left(N_{1} F\right)=$ $=(B-Q) F^{\alpha}=B G_{0} F^{\prime \prime}=B F^{1} F^{\alpha}=B F^{\alpha} ; g_{2}\left(N_{2} F^{\prime}\right)=Q F^{1}=F_{0} F^{1}=G^{\alpha} F^{1}$ und dies ist, wenn $a>1$, ein $F^{r l}$ oder $B F^{r}$. Also $g(F)=B F^{a}, g$ ist von der Klasse $0, \alpha$.

Zum Schluss wollen wir noch die topologischen Bilder von $N$ charakterisieren. Sie sind jedenfalls $G_{\delta}$ (separabel, topologisch vollständig), 0-dimensional und insichdicht (= verdichtet); aber diese Eigenschaften sind noch nicht hinreichend, da sie z.B. auch kompakten Mengen (dem Cantorschen Diskontinuum) zukommen können. Nun heisst insichdicht: es gibt keinen isolierten Punkt, d. h. keine einpunktige oder endliche offene Menge; $N$ hat aber eine darüber hinausgehende Eigenschaft:

(K) Es gibt keine kompakte offene Menge $(\neq 0)$.

Oder: jede offene Menge $G \neq 0$ enthält eine Folge $a_{l}$ ohne Häufungspunkt in $G$; in der Tat bilden im Intervall $N_{l_{1} \ldots l_{k}}$ irgendwelche Punkte $a_{l} \in N_{l_{1} \ldots l_{k} l}$ eine solche ${ }^{1}$ ), da sie paarweise die Entfernung $1 /(k+1)$ haben. Die Eigenschaft $K$ geht auch auf die mit $N$ homöomorphen Mengen über und ist nun mit den zuvor angegebenen zusammen auch hinreichend:

Die mit dem Nullraum $N$ homöomorphen Mengen sind identisch mit den separablen, topologisch vollständigen, 0-dimensionalen Räumen $X$, die keine kompakte offene Menge $(\neq 0)$ enthalten.

1) Sogar olme Häufungspunkt in $N$; auch $\bar{G}$ ist nicht kompakt; die kompakten Mesingen sind in $N$ nirgendsdicht. 
Beweis der Hinlänglichkeit ${ }^{1}$ ). Ein separabler 0-dimensionaler Raum $X$ hat eine abzählbare Basis, aus offenen und zugleich abgeschlossenen Mengen $U_{l}$ von Durchmessern $<\delta$ bestehend; es ist $X=\Sigma U_{l}$ oder, mit $D_{l}=U_{l}-\left(U_{1}+\ldots+U_{l-1}\right), X=\Sigma D_{l}$ mit disjunkten, gleichfalls offenen und abgeschlossenen Summanden $D_{l}$ von Durchmessern $<\delta$. Es kommt nun wieder darauf an, alle (oder unendlich viele) $D_{l} \neq 0 \mathrm{zu}$ erhalten; dies ist unmöglich oder möglich, je nachdem $X$ kompakt ist oder nicht. Wenn nämlich $X$ nicht kompakt ist und also eine Folge $a_{h}$ (paarweise verschiedener Punkte) ohne Häufungspunkt enthält, so gebe man jedem $a_{h}$ eine zugleich offene und abgeschlossene Umgebung $D_{h}$ vom Durchmesser $<\delta / h$ derart, dass die $D_{h}$ disjunkt sind; $D=\Sigma D_{h}$ ist offen und, wie leicht zu sehen, auch abgeschlossen; $X-D=\Sigma D_{k}$ in disjunkte (endlich oder unendlich viele) $D_{k}$ von Durchmessern $<\delta$ zerlegbar, die in $X-D$, also in $X$ offen und abgeschlossen sind, und $X=\Sigma D_{h}+\Sigma D_{k}=\Sigma D_{l}$ leistet das Verlangte. Hat $X$ die Eigenschaft $\mathrm{K}$, so ist $D_{l}$ nicht kompakt und $D_{l}=\sum_{m} D_{l_{m}}$ mit lauter disjunkten $D_{l_{m}} \neq 0$ (von beliebig kleinen Durchmessern), die in $D_{l}$, also in $X$ offen und abgeschlossen. sind. So gelangen wir zu

$$
X=\sum_{l_{1}} D_{l_{1}}, \quad D_{l_{1}}=\sum_{l_{2}} D_{l_{1} l_{2}}, \quad \ldots,
$$

$D_{l_{1} \ldots l_{k}} \neq 0$ von Durchmessern $<1 / k ;$ ist $X$ nun noch vollständig, so gibt (wegen der Abgeschlossenheit der $D$ ) $x=D_{l_{1}} D_{l_{1} l_{2} \ldots}=f(\lambda)$ eine schlichte stetige Abbildung von $N$ auf $X$, bei $\operatorname{der} f\left(N_{l_{1} \ldots l_{k}}\right)=D_{l_{1} \ldots l_{k}}$ offen und demnach jedes $f(G)$ offen ist, also eine Homöomorphie.

1) Herr Kuratowski hat mir einen kürzeren Beweis auf Grund des Satzes (6) von Herrn Mazurkiewicz mitgeteilt.

\section{Sur les ensembles plans localement connexes.}

Par

\section{Samuel Eilenberg (Warszawa).}

1. Le but de cette note est d'établir le suivant

Thérème. Si un ensemble plan, borné, connexe et localement connexe ne coupe ${ }^{1}$ ) pas le plan, il est un semicontinu ${ }^{2}$ ).

L'hypothèse que l'ensemble ne coupe pas le plan est essentielle, comme le montre un exemple de MM. Knaster et $\mathrm{Ku}$ ratowski ${ }^{3}$ ), où un ensemble plan borné, connexe et localement connexe ne contient aucun ensemble parfait, donc à plus forte raison aucun continu.

2. $S_{2}$ désignant le plan des nombres complexes, complété par le point $\infty$, et $S_{1}$ l'ensemble des points $z$ de $S_{2}$ tels que $|z|=1$, soit pour tout $z \in S_{2}-(0)-(\infty)$

$$
r(z)=z /|z| \text {. }
$$

$X$ étant un espace métrique quelconque, $S_{1}^{X}$ désigne la classe des transformations continues de $X$ en sous-ensembles de $S_{1}$. Ainsi des transformations continues de
p. ex. $\quad r \in S_{1}^{S_{2}-(0)-(\infty)}$. Etant donnée une fonction $f \in S_{1}^{X}$, nous écrirons

$$
f \sim 1 \text { sur l'ensemble } Y
$$

lorsqu'il existe une fonction $\varphi$ à valeurs réelles, définie pour tout $x \in Y$, continue et telle que $f(x)=e^{i \varphi(x)}$ pour tout $\left.x \in Y^{4}\right)$.

1) On dit que $Y$ coupe $X$ entre les points $x_{1}, x_{2} \in X$, lorsque $X-Y$ ne . On dit que $Y$ ne coupe pas $X$, lors contient aucun continu $K$ tel que $x_{1}, x_{2} \in x_{1}, x_{2} \in X-Y$.

2) $X$ 'appelle un semicontinu, lorsqu'il existe, pour tout couple $x_{1}, x_{2} \in X$, an continu $K \subset X$ tel que $x_{1}, x_{2} \in K$.

8) Bull. Amer. Math. Soc. 1927, p. 106.

4) cf. S. Filenberg, Transformations continues en circonférence et la topologie du plan, Fund. Math. 26 (1936), p. 63. 